\title{
KNOTS PRIME ON MANY STRINGS \\ BY
}

STEVEN A. BLEILER

\begin{abstract}
A study is made of the factorization of prime knots into tangles. Several infinite families of knots which do not factor into prime tangles are examined, and a new characterization of knot primality is developed.
\end{abstract}

This paper examines the factorization of knots into tangles. The notion of tangle was first introduced by J. H. Conway [3] in order to classify knots of small crossing number and to provide a streamlined method of computing certain algebraic knot invariants. F. Bonahon and L. Siebenmann [2] have given a factorization of simple knots into pieces which are either "algebraic" in a certain sense or are " $\pi$-hyperbolic". Further, R. Kirby and W. B. R. Lickorish [6, 7] have offered the notion of prime tangle; intuitively, tangles from which one can construct prime knots. We present here several infinite families of prime knots which do not factor into prime tangles.

In the first section we review the definitions and theorems of tangle theory and put forward the notion of string primality. Although our main concern is the classical situation of two strings, our definitions are phrased to apply to higher string numbers. In the second section several families of knots with high degrees of string primality are given; in $\S 3$ it is shown that for certain forms of satellite knots, the string primality of the satellite is characterized by the primality of the companion.

The author is indebted to C. McA. Gordon and W. B. R. Lickorish for many helpful discussions and to D. Seal for the explanation of his construction of 2-fold branched covers for the $K(p / q)$ knots of $\$ 2$. The reader is referred to Rolfsen's book [13] for the standard defintions and results of knot theory. Throughout this work a knot is a link of one component and all work is done in the PL category.

1. Definitions and enabling lemmas. A tangle $(B, t)$ is a 3-ball $B$ with a finite number of properly imbedded disjoint spanning arcs $t$. These arcs are called strings. (Some two-string examples are shown in Figure 1.) The ball $B$ will be omitted from most tangle diagrams. A tangle, such as Figure 1(i), is called a trivial tangle and a homeomorph of a trivial tangle, such as Figure 1(ii), is called an untangle. Conway [3] calls two-string untangles rational, as to a two-string untangle he can associate a rational number, possibly $1 / 0$, which characterizes the untangle diagram up to ambient isotopy fixing $\partial t$. Some examples appear in Figure 2. The reader is advised that the sign convention used here is opposite to that found in [3].

Received by the editors May 17, 1982 and, in revised form, March 28, 1983.

1980 Mathematics Subject Classification. Primary 57M25; Secondary 57N10, 57M12.

Key words and phrases. Prime knot, tangle, irreducible 3-manifold, string primality of knots. 
)

(i)

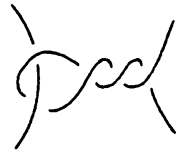

(ii)

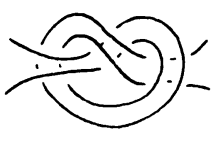

(iii)

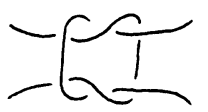

(iv)

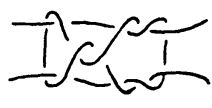

(v)

Two-string Tangles

Figure 1

)(

$1 / 0$

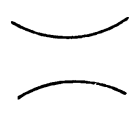

$0 / 1$

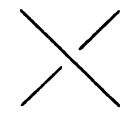

$+1$

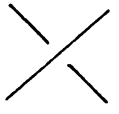

$-1$

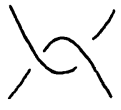

$+2$

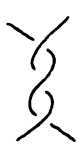

$-\frac{1}{3}$

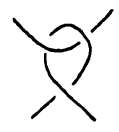

$2 / 3$

Rational Tangles

Figure 2
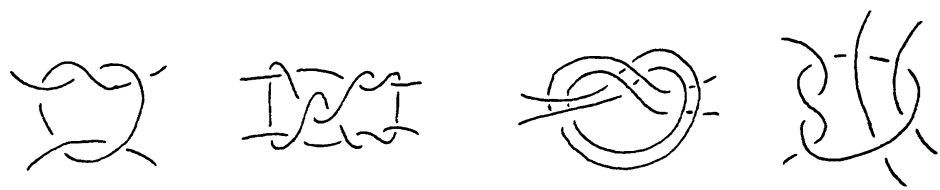

Prime Tangles

Figure 3
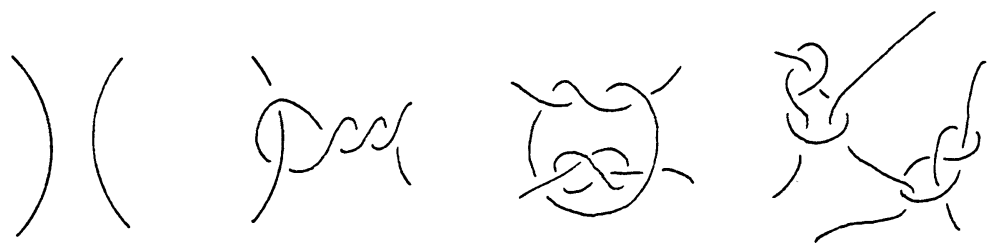

Tangles Which are Not Prime

Figure 4

A tangle $(B, t)$ is prime if:

(1) Any 2-sphere imbedded in $B$ which meets the strings transversely in two points bounds a 3-ball in $B$ which meets $t$ in a single unknotted arc.

(2) Any properly imbedded disc $D$ which meets $t$ transversely in a single point is such that $\partial D$ bounds a disc in $\partial B$ which also meets the strings in a single point.

(3) No properly imbedded disc separates the strings.

Definition. A prime knot $K$ in the 3 -sphere is an $n$-string composite if there is an imbedded 2-sphere intersecting the knot transversely which separates $\left(S^{3}, K\right)$ into prime $n$-string tangles. If $K$ is not an $n$-string composite, $K$ is said to be prime on $n$-strings. A knot which is prime on two strings is said to be doubly prime. By convention we will, on occasion, call a composite knot a 1-string composite and say that a prime knot is prime on one string.

LeMma 1.1. A prime knot $K$ in $S^{3}$ is doubly prime if and only if the exterior of $K$ does not contain a properly imbedded, incompressible, d-incompressible, quadruply punctured 2-sphere with boundary components isotopic to meridians. 
Proof First suppose that $K$ is not doubly prime; that is, a 2-sphere $F^{\prime}$ exists in $S^{3}$ separating $\left(S^{3}, K\right)$ into prime tangles $\left(A, K_{A}\right)$ and $\left(B, K_{B}\right)$. Let $E_{A}$ and $E_{B}$ denote the complement of a regular neighborhood of $K_{A}$ in $A$ and $K_{B}$ in $B$ respectively, and let $F=F^{\prime} \cap E_{A}=F^{\prime} \cap E_{B}=F^{\prime} \cap \operatorname{Ext}(K)$. By property (3) and the disc theorem $F$ is incompressible. If $F$ is $\partial$-compressible in $\operatorname{Ext}(K)$, there is a 2-disc $D$ in $A$, say, with $\partial D=\alpha \cup \beta, \alpha$ an arc in $F$ and $\beta$ an arc in $\partial E_{A}-\operatorname{Int}(\partial A)$, not isotopic rel endpoints to a curve in $\partial A$. As $\partial E_{A}-\operatorname{Int}(\partial A)$ is two cylindrical tubes parallel to the strings of $K_{A}, \beta$ runs from one end of a tube to the other. We can then form a disc which separates the strings of $A$ by taking the sides of a regular neighborhood of $D$ and the complement of this neighborhood in the tube containing $\beta$, contradicting the primality of $\left(A, K_{A}\right)$. Thus $F$ is $\partial$-incompressible.

Conversely, let $F$ be a surface as in the statement of the lemma, there is then a 2-sphere $F^{\prime}$ separating $\left(S^{3}, K\right)$ into tangles $\left(A, K_{A}\right)$ and $\left(B, K_{B}\right)$. In the notation above, both $E_{A} \cap \partial A$ and $E_{B} \cap \partial B$ are incompressible, so property (3) is satisfied. Property (2) is immediate as $\left(A, K_{A}\right)$ and $\left(B, K_{B}\right)$ are two-string tangles. If property (1) fails for $A$, say, there is a 2-ball $A^{\prime}$ in $A$ meeting $K_{A}$ in a knotted arc. Because $K$ is a prime knot, we obtain the unknot if this knotted arc is replaced by an unknotted arc. However, Van Kampen's theorem and the disc theorem together say that whenever the unknot is decomposed into tangles, at least one of the tangles must contain a disc separating the strings. This property is not affected by the replacement of an unknotted segment of a string by a knotted one. Thus either $\left(A, K_{A}\right)$ or $\left(B, K_{B}\right)$ contains a disc separating the strings. But such a disc compresses $F$, a contradiction.

As in the case of knot primality, there is a natural interpretation of string primality in terms of two-fold branched coverings. In [7] Lickorish examines the two-fold covering of the 3-ball branched over the strings of a tangle $T$, which we will denote by $M(T)$. It is shown that $M(T)$ is an irreducible, $\partial$-irreducible 3-manifold if and only if $T$ is a prime tangle. A straightforward corollary of this characterization is

LEMMA 1.2. A prime knot $K$ in the 3-sphere is an $n$-string composite $(n \geqslant 2)$ if and only if $M(K)$, the two-fold cover of $S^{3}$ branched over $K$, contains an incompressible closed surface $F$ satisfying the following conditions:

(1) $F$ is orientable of genus $n-1$.

(2) $F$ is invariant under the action of the nontrivial covering translation $\tau$ and meets the fixed point set of this map in precisely $2 n$ points.

(3) F separates $M(K)$ into irreducible, d-irreducible pieces.

In particular, Lemma 1.2 says that knots whose double-branched cover is an irreducible, nonsufficiently large 3-manifold is prime on $n$-strings for every positive integer $n$. We give specific examples in the next section.

2. Knots prime on many strings. Here we present several infinite families of doubly prime knots and knots which are prime on any number of strings. We start with some easy propositions.

THEOREM 2.1. Torus knots are doubly prime. 


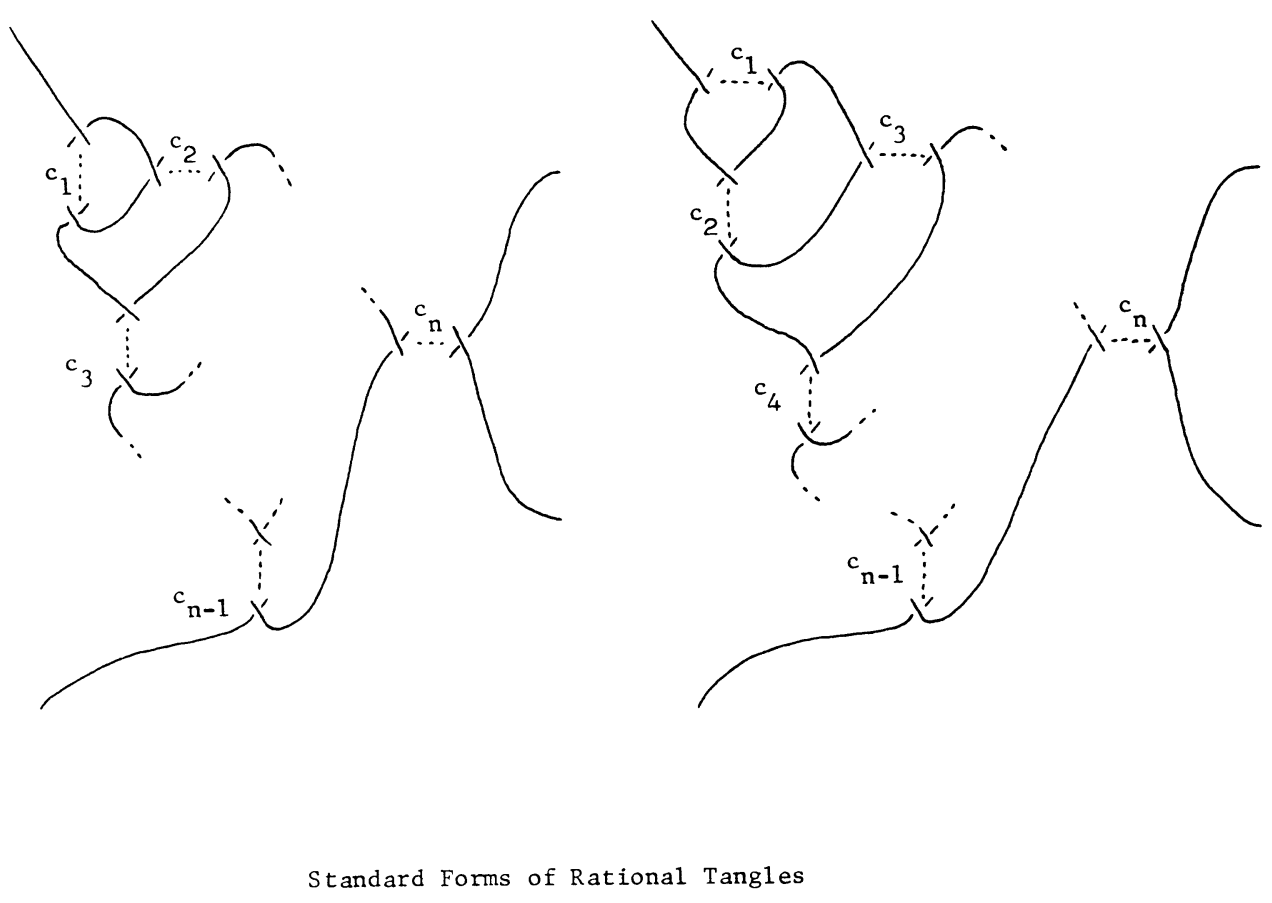

FIGURE 5

Proof. Suppose there is a torus knot $K$ which is two-composite. By Lemma 1.1 the exterior of $K$ contains a properly imbedded, incompressible, $\partial$-incompressible quadruply punctured 2-sphere which separates. Thus $\pi_{1}(\operatorname{Ext}(K))$ is a nontrivial free product with amalgamation. Because $K$ is a torus knot $\pi_{1}(\operatorname{Ext}(K))$ enjoys a nontrivial center, and therefore so must the amalgamating subgroup. But in this case the amalgamating subgroup is a free group on three generators, a centerless group. This contradiction establishes the proposition.

THEOREM 2.2. Two-bridge knots are prime on $n$-strings for every $n \geqslant 2$.

Proof. Conway [3] has shown that the two-bridge knots and links correspond bijectively with the Lens spaces via double-branched coverings. The fundamental group of a Lens space is finite and thus cannot contain the group of an orientable surface of positive genus as a subgroup. Because the fundamental group of a two-sided incompressible surface injects, we conclude that the two-fold branched cover of a two-bridge knot does not contain a surface satisfying the condition of Lemma 1.2. It follows that the two-bridge knots are prime on $n$-strings for every $n$.

For certain knots, the aforementioned correspondence of Conway's allows us to see the Seifert fibre structure of the double-branched cover. In particular, Conway has shown that up to an ambient isotopy fixing the boundary, an untangle may be 
put into one of the two forms in Figure 5. To such an untangle we associate the rational number $p / q$, where $p / q$ is computed by the continued fraction rule:

$$
\begin{aligned}
c_{n}+\frac{1}{c_{n-1}+\quad} & \\
& \frac{1}{c_{2}+\frac{1}{c_{1}}}
\end{aligned}
$$

See the examples in $\S 1$.
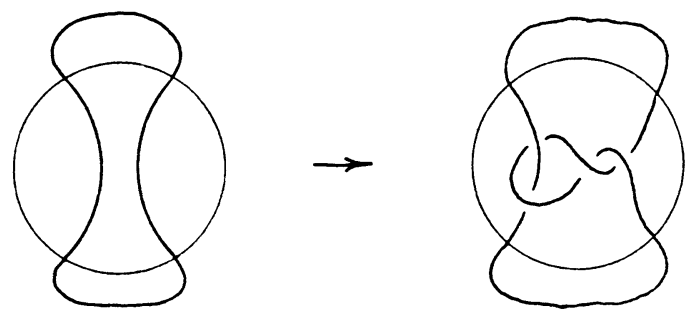

5/2 Untangle Surgery on the Unknot

FIGURE 6
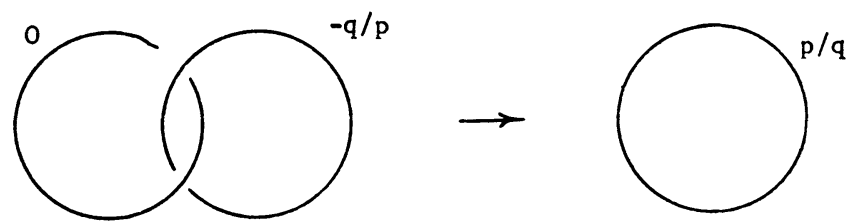

FIGURE 7
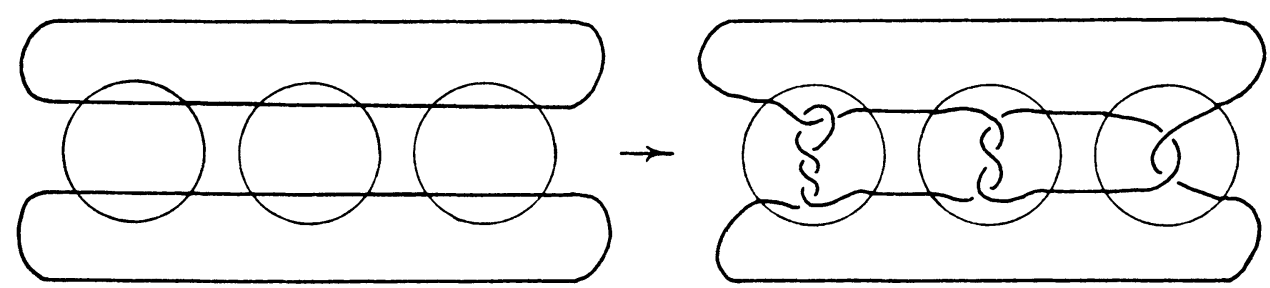

$P(7 / 2,3,-2)$

FIGURE 8 
The number $p / q$ has a natural interpretation in the double-branched cover. The double-branched cover of an untangle is a solid torus, so we can consider the Dehn surgery description of $L(p, q)$ as the left on an untangle surgery description of a two-bridge knot in $S^{3}$. A two-bridge knot is obtained when we replace a $1 / 0$ untangle in the unknot in $S^{3}$ by a $p / q$ untangle, as illustrated in Figure 6 for the figure-eight knot. As detailed in [13] or [17], this lifts to $p / q$ Dehn surgery on the unknot in $S^{3}$. This is the usual surgery description of $L(p, q)$.

In order to express $L(p, q)$ as a Seifert fibre space over $S^{2}$ with a single exceptional fibre, we must be able to obtain $L(p, q)$ by surgering a single fibre of $S^{2} \times S^{1}$, taking care not to kill the fibre of $S^{2} \times S^{1}$ homotopically. The coefficient of this surgery is given in the following lemma.

LemMA 2.3. The Lens space $L(p, q)$ is obtained by performing $-q / p$ surgery on $a$ fibre of $S^{2} \times S^{1}$.

Proof. The 3-manifold obtained by performing $-q / p$ surgery on a fibre of $S^{2} \times S^{1}$ can be pictured in the Kirby-Rolfson calculus [11] as surgery on a Hopf link in $S^{3}$, as pictured in Figure 7. The complement of a regular neighborhood of the component labeled $-p / q$ is a solid torus with 0 -surgery performed on the core. This surgery results in a solid torus but changes a longitude to a meridian and a meridian to the negative of a longitude. The Hopf link description is thus equivalent to $p / q$ surgery on the unknot.

Summarizing, via double-branched coverings, surgery on a fibre of $S^{2} \times S^{1}$ which does not kill the homotopy of the fibre may be viewed as the lift of the removal of a $0 / 1$ untangle from the unlink in $S^{3}$ as illustrated in Figure 8 and its replacement by an untangle other than the one corresponding to $1 / 0$. Further, the surgery coefficient in $S^{2} \times S^{1}$ and the rational number characterizing the replacement untangle are negative reciprocals.

A (3-braid) rational pretzel knot which has a projection obtained from the unlink by exactly three untangle surgeries, as illustrated in Figure 8 . Given rational numbers $r_{1}, r_{2}, r_{3}$ we denote by $P\left(r_{1}, r_{2}, r_{3}\right)$ the rational pretzel knot or link obtained by replacing three copies of a $0 / 1$ untangle by the untangles corresponding to $-r_{i}^{-1}$, $1 \leqslant i \leqslant 3$. This is illustrated for $P(7 / 2,3,-2)$, the knot $10_{132}$ in the table of Rolfsen and Bailey [13]. Up to ambient isotopy, the knot or link obtained by this construction is independent of the order of the $r_{i}$; however, this is not true in the more general case in which more than three untangle surgeries are performed.

THEOREM 2.4. The (3-braid) rational pretzel knots are prime on $n$ strings for every $n \geqslant 2$.

Proof. The arguments above show that $M_{P}$, the double-branched cover of the rational pretzel knot $P=P\left(r_{1}, r_{2}, r_{3}\right)$, is obtained from $S^{2} \times S^{1}$ by surgering three distinct fibres $f_{1}, f_{2}, f_{3}$ according to the instructions $r_{1}, r_{2}, r_{2}$ respectively. The manifold $M_{P}$ thus has the structure of a Seifert fibre space over $S^{2}$ with no more than three exceptional fibres. The Seifert fibre structure on the double-branched cover of a rational pretzel knot was noticed and extensively studied by J. M. 
Montesinos $[9,10]$. If the Seifert fibre structure on $M_{p}$ has less than three exceptional fibres, $M_{p}$ is a Lens Space [5]; and as in the proof of Theorem 2.2 we are done. On the other hand, Waldhausen [20] has shown that a Seifert fibre space over $S^{2}$ with exactly three exceptional fibres does not contain a separating incompressible surface (and hence has no incompressible surfaces if the first homology is finite). So we may again apply Lemma 1.2 to conclude that the rational pretzel knots are prime on $n$-strings for every $n \geqslant 2$.

REMARKS. Using this correspondence between rational pretzel knots and Seifert fibre spaces over the 2-sphere J. Birman, F. Gonzalez-Acuña, and J. Montesinos [1] showed that the same 3-manifold may arise as the double cover of $S^{3}$ branched over nonisotopic knots. In particular, the Brieskorn manifold $\Sigma(2, p, q)$ is the double cover of $S^{3}$ branched over the $(p, q)$ torus knot. If both $p$ and $q$ are different from 2 , $\Sigma(2, p, q)$ has the structure of a Seifert fibre space over $S^{2}$ with three exceptional fibres and is thus the double-branched cover of a rational pretzel knot. With a finite number of exceptions, for example the $(3,4)$ torus knot is $P(-2,3,3)$, these knots are distinct and can be distinguished by their Conway potentials. This phenomena also occurs in our next example.

By classifying the incompressible, $\partial$-incompressible surfaces in the figure-eight knot complement, W. Thurston [19] gave new examples of irreducible, nonsufficiently large 3-manifolds with infinite fundamental group. He shows that among the 3-manifolds obtained by performing Dehn surgery on the figure-eight knot only those obtained from 0 or \pm 4 surgery are sufficiently large. Thruston also shows that the manifold obtained from $p / q$ surgery on the figure-eight knot is hyperbolic, i.e. admits a complete Riemannian metric with constant, negative sectional curvature, whenever $|p|>4$ and $|q|>1$; thus these manifolds cannot admit a Seifert fibre structure.

Now consider the family $K(p / q)$ constructed as illustrated in Figure 9, where the $p / q$ untangle is inserted in the space labeled $p / q$. A knot or link with a projection as in this family is called a $K(p / q)$ knot or link respectively. Observe that $K(p / q)=$ $K(-p /-q)$ and that if $|q|=1, K(p / q)$ is a knot or link according to $p$ odd or even.

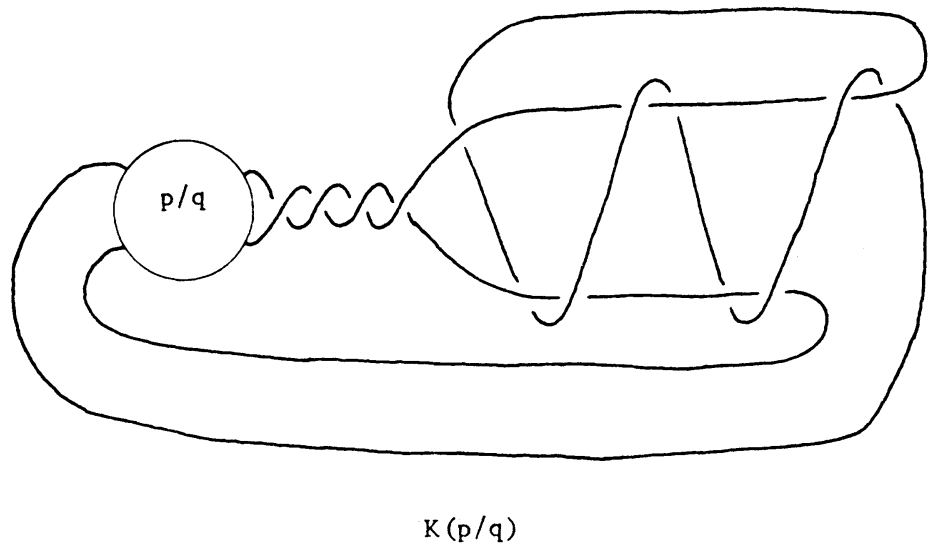

FIGURE 9 
THEOREM 2.5. $A K(p / q)$ knot is prime on $n$-strings for every $n \geqslant 1$.

Proof. As $K(0 / 1), K(4 / 1)$, and $K(-4 / 1)$ are links, the theorem follows from the remarks above and

LEMMA 2.6. The double cover of $S^{3}$ branched over the knot $K(p / q)$ is obtained from $p / q$ surgery on the figure-eight knot.

Proof. One proves this lemma by applying the technique of untangle surgery.

As previously observed, Conway notes in [3] that the 3-manifold obtained by performing $p / q$ Dehn surgery on an unknot double covers the 3-sphere branching over the knot or link obtained by replacing a $1 / 0$ untangle by a $p / q$ untangle in a suitable projection of the unknot.

One can use the same idea to see how the manifold obtained by Dehn surgery on the figure-eight knot double-branch covers the 3-sphere. In order to correctly perform the untangle surgery, one must keep track of the "framing", that is, one must know the preferred latitude of the untangle. The isotopy class of this curve is obtained by projecting both the preferred longitude and its translate under the deck transformation to the boundary of the untangle to be surgered. For the figure-eight knot this is given in Figure 10.

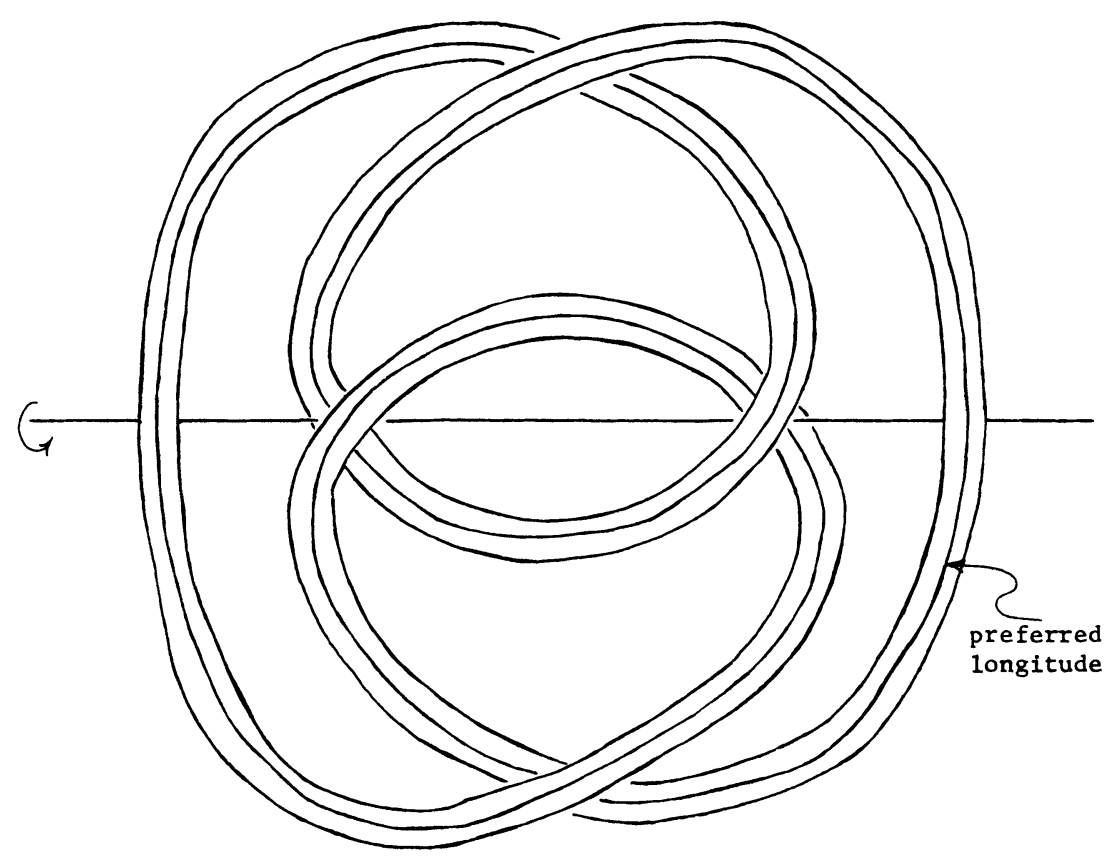

FIGURE 10 


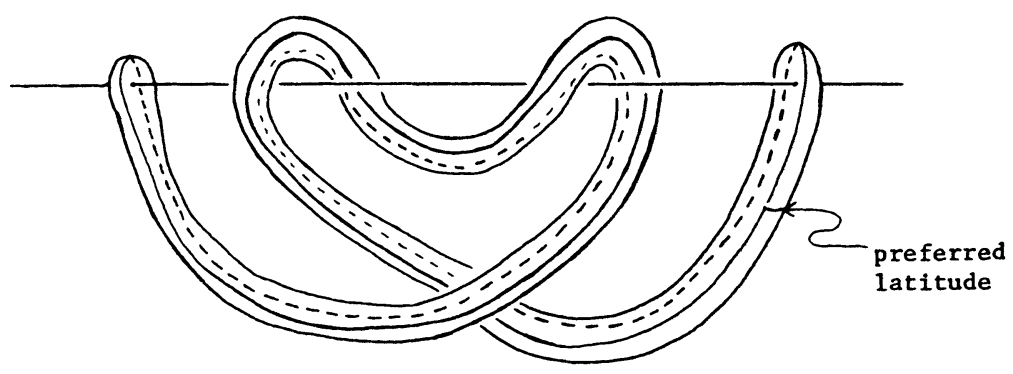

$\downarrow$ isotopy

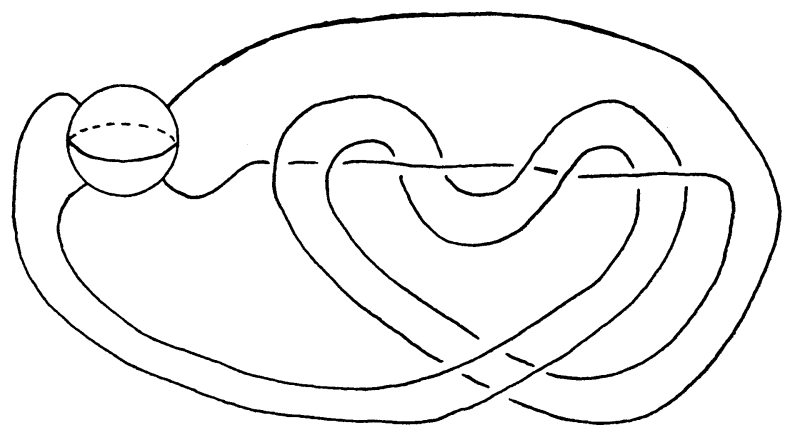

isotopy

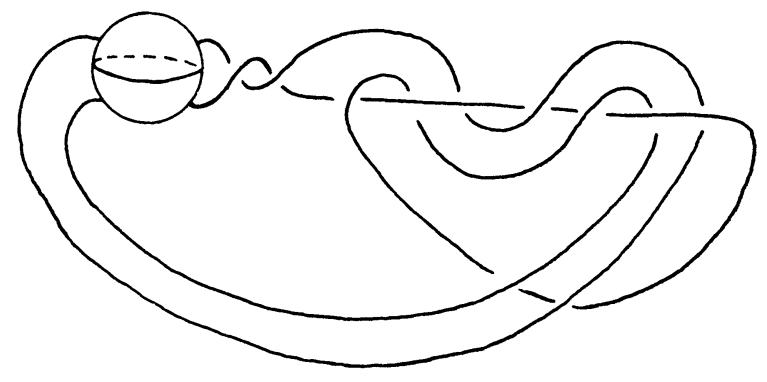

Figure 10 (continued) 

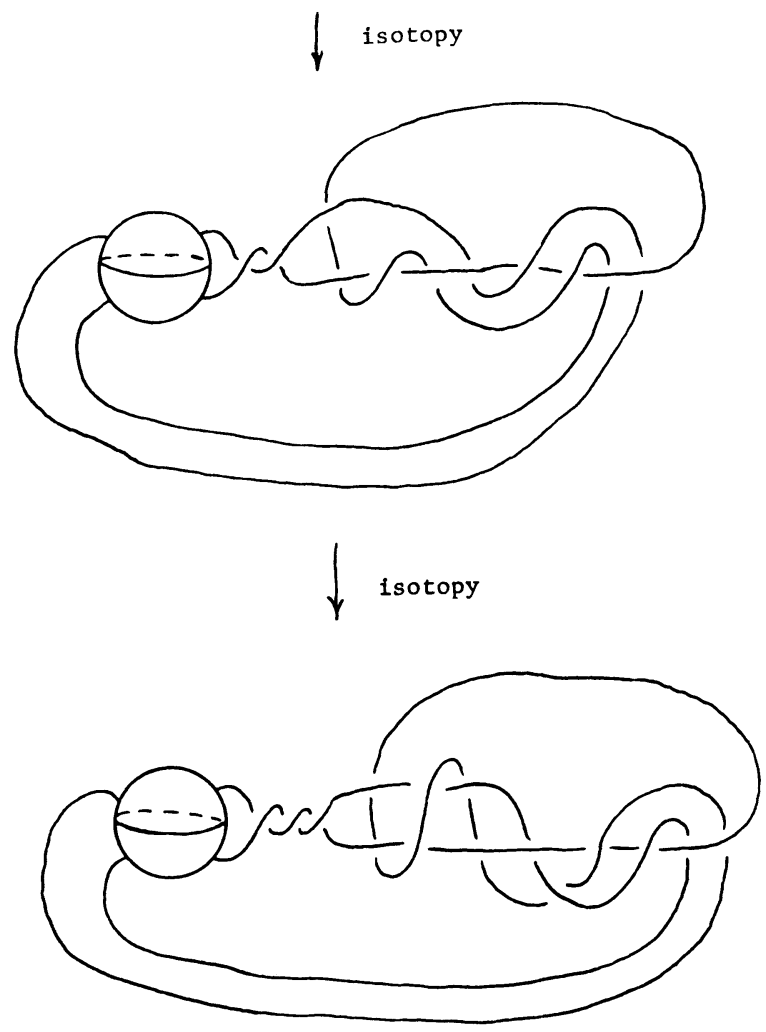

$\downarrow$ isotopy

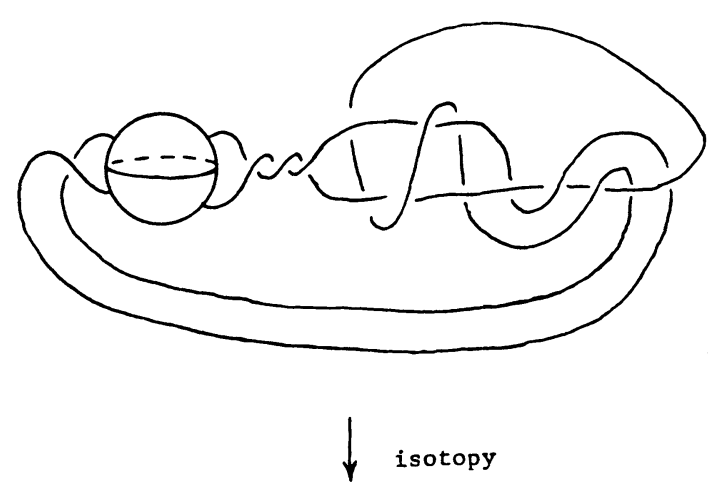

FIGURE 10 (continued) 

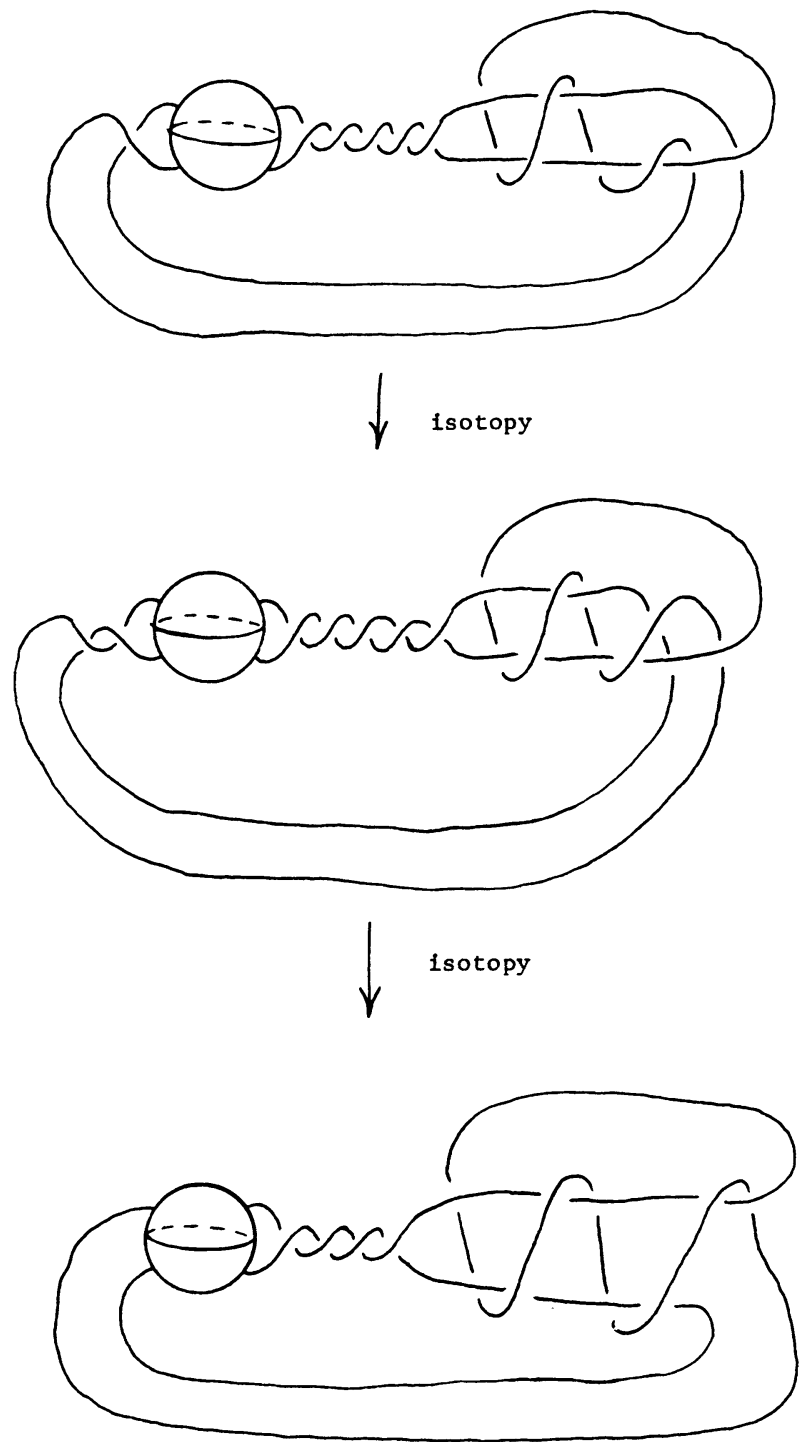

Figure 10 (continued)

REMARKS. As the figure-eight knot is amphicheiral, there is an orientation reversing homeomorphism between the manifolds obtained from $p / q$ and $-p / q$ surgery on the figure-eight knot. The double-branched covers of $K(p / q)$ and $K(-p / q)$ are thus homeomorphic. If $p \neq 0$ these knots may be distinguished by the Conway potential function and thus are nonisotopic knots with homeomorphic doublebranched covers. Indeed, $K(1 / 1)$ and $K(3 / 1)$ are the rational pretzel knots $P(-2,3,7)$ and $P(4,3,3 / 2)$ while $K(-1 / 1)$ and $K(-3 / 1)$ are the $(3,7)$ and $(3,8)$ torus knots. The $K(5 / 1)$ knot is the duplication $10_{161} \cong 10_{162}$, first observed by Perko, in the tables of both Little and Conway [12]. 
3. String primality and companionship. For a knot $K$ in the 3-sphere we form its satellites in the following manner. Take a second knot $E$ embedded in a solid torus $V$ in such a way that every meridional disc of $V$ meets $E$ nontrivially. Map $V$ homeomorphically to $V_{K}$, a tubular neighborhood of $K$, via a homeomorphism $h$ which carries a meridian of $V$ to a meridian of $V_{K}$ and the preferred longitude of $V$ to the sum of the preferred longitude of $V_{K}$ with $q$ meridians. The knot $h(E)$ is a $q$-twist satellite of $K$ with embellishment $E$. The knot $K$ is called a companion of $h(E)$, the torus $\partial V_{K}$ is called a comparion torus. $H$. Schubert [15] has shown that if $K$ is nontrivial and every meridional disc of $V$ meets $E$ in at least two points and if the pair $(V, E)$ does not contain a knotted ball-arc pair then the knot $h(E)$ is prime.

An important class of satellite knots are the $q$-twist doubles, formed when the embellishment is an unknot imbedded in a solid torus as illustrated in Figure 11. A generalized double is obtained when an unknot is imbedded as in doubles but with more than two half-twists. For generalized doubles there is a close relationship between the primality of the companion and the string primality of the knot.

THEOREM 3.1. A knot $K$ in the 3-sphere is prime if and only if any generalized double of $K$ is doubly prime.

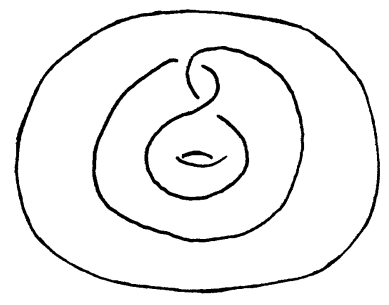

A Doubling Curve

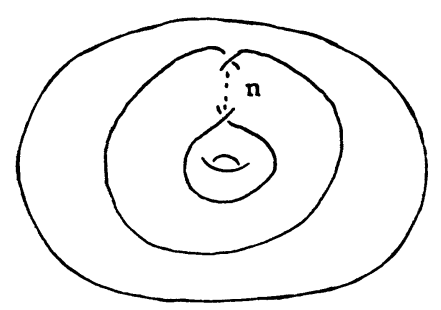

A Generalized Doubling Curve

FIGURE 11
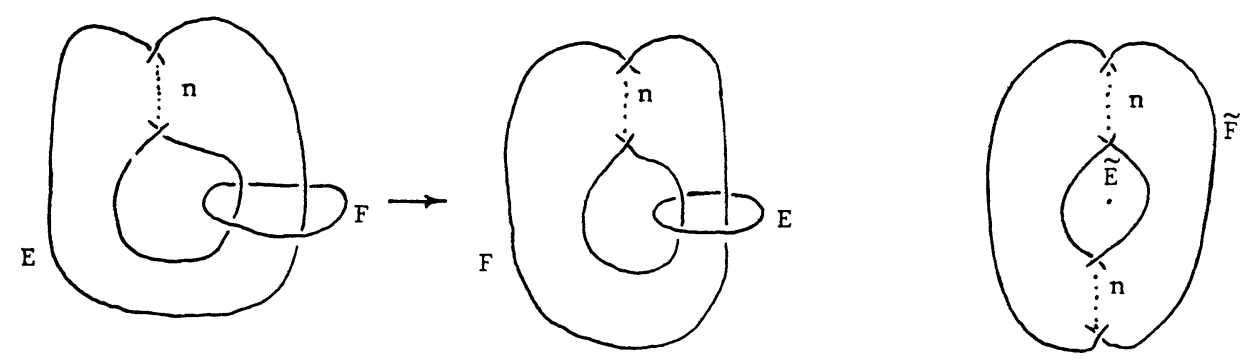

A Torus Link Exterior Double-covering V 
Proof. Let $D_{K}$ be a generalized double of $K$. The proof follows from Lemma 1.2 and an analysis of the incompressible tori in $M\left(D_{K}\right)$, the double-branched cover. For convenience denote this space by $M$.

The companion torus $\partial V_{K}$ lifts to two disjoint incompressible tori in $M$ and cutting $M$ along these tori we obtain three pieces. Two are copies of the exterior of $K$. The third component, call it $L$, is the double cover of the solid torus branched over the embellishment $E$. The space $L$ can be obtained by considering the 3 -sphere as the double cover of itself branched over the unknot $E$. As illustrated in Figure 12, the solid torus $V$ in the construction of $D_{K}$ can be thought of as the exterior of an unknotted curve $F$. We obtain $L$ by removing the lifts of this neighborhood, displaying $L$ as a link exterior. To determine this link, observe that $E$ and $F$ are symmetric; that is, there is an ambient isotopy of $S^{3}$ interchanging the positions of $E$ and $F$. By viewing $E$ as the $z$-axis we see that $L$ is the $(2,2 n)$ torus link exterior with covering translation given by rotation through $\pi$ about the $z$-axis. The space $L$ has a natural Seifert fibre structure over the annulus with a single exceptional fibre of type $(n, 1)$.

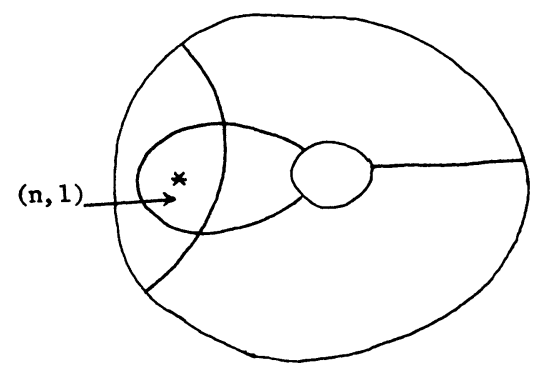

The Essential Arcs in the Orbit Manifold of $L$

FIGURE 13
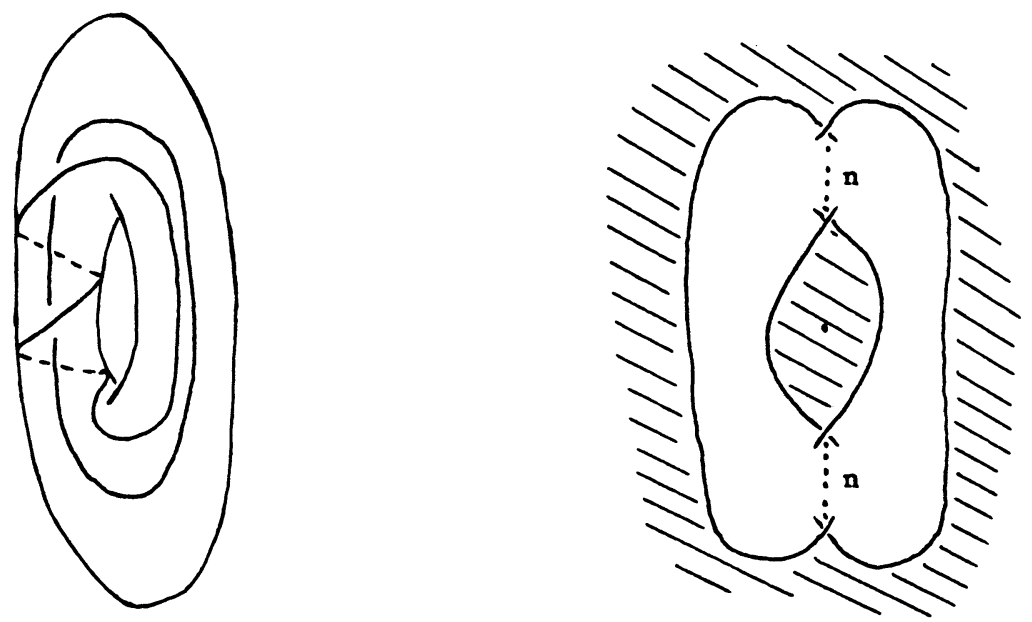

Visualizing the Essential Annulf in L

FIGURE 14 
Now suppose that $D_{K}$ is 2-composite. Further suppose that $T$, the lift to $M$ of the separating 2-sphere, has been positioned via isotopy to have minimal transverse intersection with the lifts of the companion torus. As $T$ meets the branch set, $T \cap L$ is nonempty. The torus $T$ is not entirely contained in $L$, for in any Seifert fibre space over an annulus with a single exceptional fibre an incompressible torus is boundary parallel. Further, no component of $T \cap L$ or $T \cap$ \{knot exteriors $\}$ is a disc as knot and link exteriors are irreducible, $\partial$-irreducible 3-manifolds and $T$ has minimal transverse intersection with the lifts of the companion torus. We conclude that $T$ meets $L$ and the knot exteriors in essential annuli.

We need to know the isotopy classes of the boundary curves of these essential annuli in the knot exteriors; as the work of J. Simon [18] shows that a knot whose exterior contains a properly embedded essential annulus is composite if the boundary components represents meridians and a cable knot if the boundary components do not. To determine these curves, we determine the essential annuli in $L$ up to isotopy. In a Seifert fibre space such as $L$ any essential annulus may be isotoped until it is either a union of fibres or transverse to all of the fibres [5]. The latter is forbidden in this case by the Euler characteristic, so the essential annuli in $L$ correspond to the essential arcs in the orbit manifold of $L$. As illustrated in Figure 13 there are three such arcs up to isotopy.

The essential annuli corresponding to these arcs are easily visualized. Two are annuli with both boundary components on a single peripheral torus. These annuli can be visualized by placing one component of the $(2,2 n)$ torus link on the boundary of a solid torus and the other component inside. The third essential annulus has a boundary component on each peripheral torus. As illustrated in Figure 14, this is the Seifert annulus for the $(2,2 n)$ torus link if we orient this link by lifting the orientation for the curve $F$. This annulus is also the lift of a meridional disc of $V$. Note that with this choice of orientations, the $(2,2 n)$ torus link is not fibred.

Of the three annuli, only the Seifert annulus intersects the branch set and therefore is a component of $T \cap L$. By mapping a boundary component of the Seifert annulus via the lift of the restriction of $h$ to $\partial V$ we obtain a curve $C$ on the peripheral torus of the exterior of $K$ which bounds an essential annulus. As the Seifert annulus is the lift of a meridional disc of $V$, the curve $C$ must represent a meridian of $K$. Thus $K$ is a composite knot.

Conversely, if $K$ is a composite knot, the exterior of $K$ contains an essential annulus with boundary components meridians. In $M$ we obtain an incompressible torus as in Lemma 1.2 from two copies of the Seifert annulus and a copy of the aforementioned annulus in each knot complement. Thus $D_{K}$ is 2-composite.

The above theorem tempts one to conjecture a relation between the string primality of a satellite, the string primality of both the embellishment and the companion, and the geometric winding number. This does not seem to be the case, however, As we illustrate in Figure 15, there also are satellites of the trefoil with embellishment the unknot and geometric winding number two which are 2-composite. 


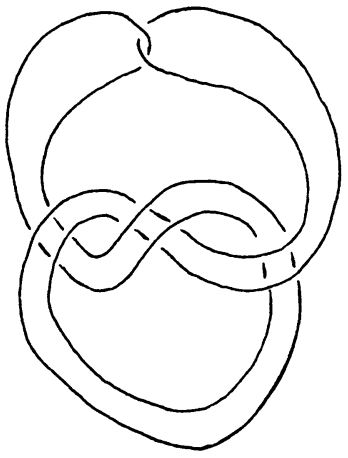

Doubly Prime

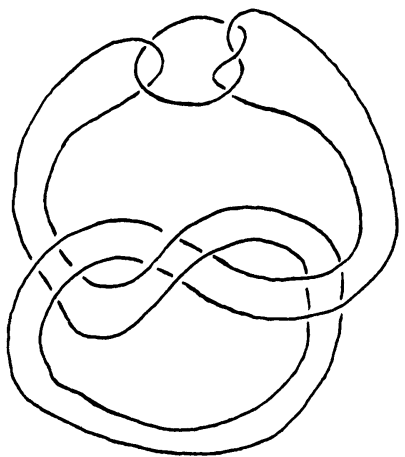

Two-Compos 1 te

Satellites of the Trefoll with Embellishment the Unknot

FIGURE 15

4. Concluding remarks. The group of a tame knot in the 3-sphere has a presentation in which the number of relators is one less than the number of generators, but no presentation with two fewer relators than generators. Algebraically, the "simplest" knots are thus the ones with a presentation with a single relator. A one-relator knot is also a two-generator knot, these latter knots possibly form a more general class as a knot could have the property that any two-generator presentation of its group requires at least two relators, see [4]. The best known examples of two-generator knots are the two-bridge and torus knots. The two-generator knots were shown to be prime by F. Norwood [11] and the natural conjecture is that two-generator knots are doubly prime.

Progress on this conjecture has been recently achieved by M. Scharlemann [14]. He examines the knots with the property that a 1-cell may be attached to the knot so that the complement of a regular neighborhood of the result is a standard genus two handlebody. Such a knot is said to have tunnel number one. The class of tunnel number one knots has been conjectured to coincide with the class of one relator knots but this conjecture remains unsettled. Scharlemann shows that tunnel number one knots are doubly prime, thus giving another method of establishing the string primality of a knot.

An additional technique to determine string primality exists for the alternating knots. W. Menasco [8] has given an algorithm for locating the incompressible, d-incompressible surfaces in the complement of an alternating knot or link. In particular, he shows that a planar surface as in Lemma 1.1 can meet the projection plane of an alternating projection of a knot in precisely two ways. A sphere separating an alternating knot into prime tangles is thus either "obvious" or else meets the projection plane of an alternating projection in exactly two circles, configured in a way first pointed out by Conway and occurring most simply in the Borromean rings. As an example, Figure 17 shows how the configuration occurs in the knot $8_{16}$ of the table of Alexander and Briggs. In principal, this gives an algorithm for determining the 2-string primality of an alternating knot. 


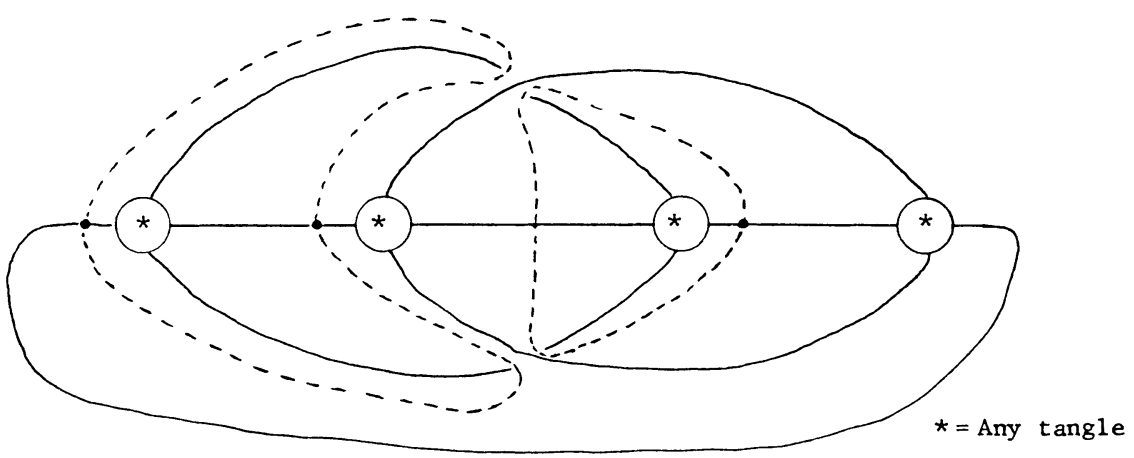

Alternate Configuration of a Separating Sphere

FigURE 16
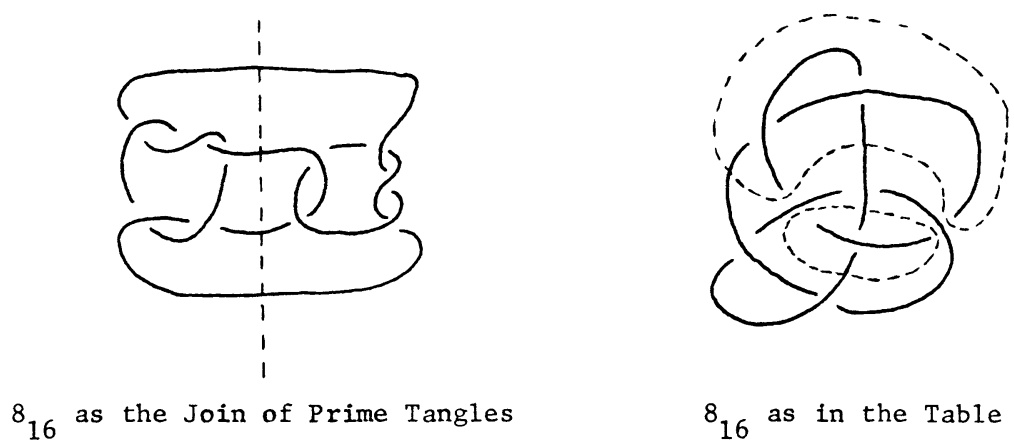

${ }_{16}$ as in the Table

FIgURE 17

\section{REFERENCES}

1. J. Birman, F. Gonzalez-Acuña and J. Montesinos, Heegard splittings of prime 3-manifolds are not unique, Michigan J. Math. 23 (1976), 97-103.

2. F. Bonahon and L. Siebenmann, New geometric splittings of classical knots (to appear).

3. J. H. Conway, An enumeration of knots and links, and some of their algebraic properties, Computational Problems in Abstract Algebra, Pergamon Press, Oxford and New York, 1969, pp. 329-358.

4. M. J. Dunwoody and A. Pietrowski, Presentations of the trefoil group, Canad. Math. Bull. 16 (1973), $517-520$.

5. W. Jaco, Lectures on 3-manifolds topologv, CBMS Regional Conf. Ser. in Math., no. 43, Amer. Math. Soc., Providence, R. I., 1979.

6. R. Kirby and W. B. R. Lickorish, Prime knots and concordance, Math. Proc. Cambridge Philos. Soc. 86 (1979), 437-441.

7. W. B. R. Lickorish, Prime knots and tangles, Trans. Amer. Math. Soc. 267 (1981), 321-332.

8. W. Menasco, Incompressible surfaces in the complement of alternating knots and links (preprint).

9. J. M. Montesinos, Variedas de Seifert que non recubridores eiclicos ramificados de dos hojas, Bol. Soc. Mat. Mexicana 18 (1973), 1-29.

10. _ Revêtements ramifies de noeuds espaces fibres de Seifert et scidements de Heegaard, Conferences, 1976, Orsay.

11. F. H. Norwood, One-relator knots, Ph. D. Dissertation, University of Southwestern Louisiana, 1979.

12. K. Perko, On 10 crossing knots (to appear).

13. D. Rolfsen, Knots and links, Mathematics Lecture Series, vol. 7, Publish or Perish, Berkeley, Calif., 1976. 
14. M. Scharlemann, Tunnel number one knots are doubly prime (preprint).

15. H. Schubert, Knoten und Vollringe, Acta Math. 90 (1953), 131-286.

16. D. Seal, Combinatorial double branched covers (to appear).

17. L. Siebenmann, Exercises sur les noeuds rationnels, Orsay, 1975.

18. J. Simon, An algebraic classification of knots in $S^{3}$, Ann. of Math. (2) 97 (1973), 1-13.

19. W. Thurston, The geometry and topology of 3-manifolds, Mimeographed Notes, Princeton University, 1978.

20. F. Waldhausen, Gruppen mit Zentrum und 3-dimensionale Mannigfaltigkeiten. I, II, Invent. Math. 3 (1967), 308-333; ibid. 4 (1967), 87-117.

Department of Mathematics, The University of TeXas, Austin, TeXas 78712 